\section{Protein delivery using VP22}

To the editor:

The herpes simplex virus protein VP22 has been reported to translocate between cultured mammalian cells ${ }^{1}$. VP22 expressed in cultured cells is exported and spreads to surrounding cells, where it accumulates in the nuclei. The activity of the VP22 protein can also deliver heterologous protein sequences, making it a unique and useful tool to study molecular and cellular processes.

We have developed commercially available vectors for the production of VP22 fusion proteins in mammalian cells and Escherichia coli. Like Lundberg and Johnson ${ }^{2}$, we could detect import of VP22-green fluorescent protein (GFP) fusion purified from E. coli by immunofluorescence, but had considerable difficulties detecting import by direct GFP fluorescence in either live or fixed cells. In transfected cultures, VP22-GFP protein in the nucleus could only be detected using confocal microscopy, suggesting that detection is made difficult by the low level of GFP fluorescence.

To overcome the limitation of GFP detection, we developed gene reporter assays that rely on the activity of the fusion partner to monitor VP22 delivery. Reporter assays allow detection of VP22 translocation without the need for immunofluorescence or the potential for fixation artifacts associated with these protocols.

Flp recombinase was initially chosen as a reporter to demonstrate intercellular transport of a VP22 fusion protein because a reporter assay was readily available. The reporter plasmid, pFIN4/lacZ, has a seg-
A

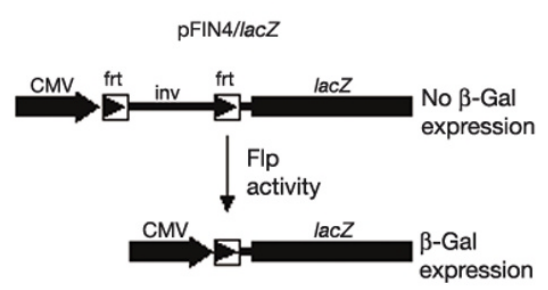

ment of DNA flanked by frt sites (recombination sites recognized by Flp) separating the cytomegalovirus (CMV) promoter and the $\beta$-galactosidase gene (Fig. 1A). Cells transfected with pFIN4/lacZ do not express $\beta$-galactosidase because a transcriptional terminator is located between the frt sites. However, in the presence of Flp recombinase the segment of DNA is removed and $\beta$-galactosidase is expressed. The gene encoding Flp recombinase was cloned into pVP22/myc-His-2 (Invitrogen, Carlsbad CA). Plasmids encoding VP22-Flp, Flp (pOG44), or VP22 alone were transfected into Chinese hamster ovary $(\mathrm{CHO})$ cells. At the same time, COS cells were transfected with pFIN4/lacZ. Twenty-four hours post transfection, each $\mathrm{CHO}$ cell transfection was mixed with a COS cell transfection. COS cells transfected with pFIN4/lacZ expressed $\beta$-galactosidase only when mixed with $\mathrm{CHO}$ cells expressing the Flp-VP22 fusion protein (Fig. 1B). CHO cells expressing VP22 or Flp recombinase alone did not activate $\beta$-galactosidase expression upon mixing cell types. This experiment demonstrates that VP22 can be used to deliver functional Flp recombinase and that VP22 can translocate between different cell types.

VP22 protein can be purified from E. coli and added exogenously to cells to deliver functional proteins ${ }^{3}$. We have successfully purified and delivered several VP22 fusion ogen). One of

Figure 1. Translocation of VP22-Flp protein fusion. (A) The reporter plasmid pFIN4/lacZ has an intervening sequence (inv) flanked by Flp recognition sites (frt) separating the CMV promoter and b-galactosidase gene (lacZ). Interaction of Flp recombinase with $p F I N 4 / / a c Z$ removes the inv sequence, resulting in $\beta$ galactosidase expression. (B) Twenty-four hours post-transfection, COS cells transfected with $\mathrm{pFIN} 4 / \mathrm{lac} Z$ were mixed with $\mathrm{CHO}$ cells expressing VP22, VP22-Flp, or Flp (pOG44) and incubated together for 24 hours. Cells were then fixed with $4 \%$ formaldhyde and stained for $\beta$-galactosidase activity using X-gal. Cells expressing $\beta$-galactosidase appear dark colored. proteins from E. coli using the Voyager Protein Production kit vector, pCRT7/ VP22 (Invitrthe first VP22 fusion proteins purified in our laboratory was with the $99 \mathrm{kDa}$ protein T7 RNA polymerase (T7 RNAP). In vitro transcription analysis determined that the

available T7 RNAP preparations. To test activity in vivo, cells were transfected with a plasmid that has the luciferase reporter under control of the T7 promoter. Luciferase activity could be detected in cells treated with the VP22-T7 RNAP fusion (Fig. 2). Furthermore, expression was dose-dependent; increasing the amount of VP22-T7 RNAP increased the overall expression levels. Maximum luciferase activity with VP22-T7 RNAP was $60 \%$ of the level observed with $\mathrm{CHO}$ cells co-transfected with T7-luc and a CMV vector expressing the unfused T7 RNAP sequence. This demonstrates that large VP22 fusions purified from E. coli can be delivered to cells in culture.

In summary, we have demonstrated VP22 delivery using reporter gene assays between cells in culture and by exogenous addition of VP22 fusion protein to cultured mammalian cells. To date, we have demonstrated VP22 import in more than 10 different mammalian and insect cell lines. We believe that these assays are more sensitive and a more relevant demonstration of VP22 translocation than delivery of the GFP reporter or immunofluorescence.

Robert P. Bennett and Brian Dalby, Research and Development, Invitrogen Corporation, 1600 Faraday Avenue, Carlsbad CA 92008 (r.p.bennett@invitrogen.com) $\mathrm{p} \mathrm{u} \mathrm{r} \mathrm{i} \mathrm{f} \mathrm{i} \mathrm{e} \mathrm{d}$ VP22-T7 RNAP fusion had activity levels comparable to commercially
1. Elliott, G. \& O'Hare, P. Cell 88, 223-233 (1997).

2. Lundberg, M. \& Johnson, M. Nat. Biotechnol. 19, $713(2001)$.

3. Kueltzo, L.A., Normand, N., O'Hare, P. \& Middaugh, C.R. J. Biol. Chem. 275, 33213-33221 (2000). 